\title{
Long-range interactions between excited helium and alkali-metal atoms
}

\author{
J.-Y. Zhang, ${ }^{1, *}$ L.-Y. Tang, ${ }^{2}$ T.-Y. Shi, ${ }^{2,3}$ Z.-C. Yan ${ }^{2,3,4}$ and U. Schwingenschlögl ${ }^{1, \dagger}$ \\ ${ }^{1}$ Division of Physical Science and Engineering, King Abdullah University of Science and Technology, Thuwal 23955-6900, Saudi Arabia \\ ${ }^{2}$ State Key Laboratory of Magnetic Resonance and Atomic and Molecular Physics, Wuhan Institute of Physics and Mathematics, \\ Chinese Academy of Sciences, Wuhan 430071, People's Republic of China \\ ${ }^{3}$ Center for Cold Atom Physics, Chinese Academy of Sciences, Wuhan 430071, People's Republic of China \\ ${ }^{4}$ Department of Physics, University of New Brunswick, Fredericton, New Brunswick, Canada E3B 5A3 \\ (Received 10 September 2012; revised manuscript received 4 November 2012; published 3 December 2012)
}

\begin{abstract}
The dispersion coefficients for the long-range interaction of the first four excited states of $\mathrm{He}$, i.e., $\operatorname{He}\left(2^{1,3} S\right)$ and $\mathrm{He}\left(2^{1,3} \mathrm{P}\right)$, with the low-lying states of the alkali-metal atoms $\mathrm{Li}, \mathrm{Na}, \mathrm{K}$, and $\mathrm{Rb}$ are calculated by summing over the reduced matrix elements of the multipole transition operators. For the interaction between $\mathrm{He}$ and $\mathrm{Li}$ the uncertainty of the calculations is $0.1-0.5 \%$. For interactions with other alkali-metal atoms the uncertainty is $1-3 \%$ in the coefficient $C_{5}, 1-5 \%$ in the coefficient $C_{6}$, and 1-10\% in the coefficients $C_{8}$ and $C_{10}$. The dispersion coefficients $C_{n}$ for the interaction of $\mathrm{He}\left(2^{1,3} S\right)$ and $\mathrm{He}\left(2^{1,3} P\right)$ with the ground-state alkali-metal atoms and for the interaction of $\mathrm{He}\left(2^{1,3} S\right)$ with the alkali-metal atoms in their first ${ }^{2} P$ states are presented in this Brief Report. The coefficients for other pairs of atomic states are listed in the Supplemental Material.
\end{abstract}

DOI: 10.1103/PhysRevA.86.064701

PACS number(s): 34.20.Cf, 34.50.Cx, 34.50.Fa

Cold and ultracold mixtures of multispecies atoms are an active topic of research in recent years. Ultracold mixtures of two atomic species are used to study the elastic and inelastic collisions between different species. Such mixtures also make it possible to sympathetically cool one species through collisional energy exchange with the other species [1-3]. In addition, weakly bound heteronuclear molecules can be created [4-6]. Heteronuclear polar molecules have attracted particular attention because their permanent dipole moments can be controlled with external fields. They are potential candidates for quantum simulation, quantum computing, and quantum metrology $[7,8]$. Most dual-species experiments involve combinations of alkali-metal atoms, such as $\mathrm{Na}-\{\mathrm{K}$, $\mathrm{Rb}, \mathrm{Cs}\}$ [9], $\mathrm{K}-\{\mathrm{Rb}, \mathrm{Cs}\}$ [2,6,9], and Rb-Cs [9,10]. Recently, a few experiments have employed ultracold mixtures of alkalimetal and metastable noble gas atoms, such as ${ }^{40} \mathrm{Ar}^{*}{ }^{87} \mathrm{Rb}$ $[11,12]$ and $\mathrm{He}^{*}{ }^{87} \mathrm{Rb}[12-14]$. The latter mixture is promising for creating a dual-species Bose-Einstein condensate by significantly suppressing the Penning ionization [13-15], which causes the trap losses. The loss rate is determined primarily by the long-range interaction between the $\mathrm{He}^{*}$ and ${ }^{87} \mathrm{Rb}$ atoms.

There are very few calculations of dispersion coefficients reported in the literature for the long-range interaction between low-lying excited states of $\mathrm{He}$ and alkali-metal atoms. The coefficients $C_{6}$ for the van der Waals interaction between the $\mathrm{He}\left(2^{1,3} S\right)$ metastable states and the ground states of $\mathrm{Li}$, $\mathrm{Na}, \mathrm{K}, \mathrm{Rb}$, and $\mathrm{Cs}$ have been computed by Bell et al. [16] by means of the Casimir-Polder formula [17]. Dalgarno and Victor [18] have improved the calculation by more accurate representations of the dynamic dipole polarizabilities $\alpha_{1}(\omega)$ of the metastable states. For the alkali-metal atoms, these authors employ semiempirical representations of $\alpha_{1}(\omega)$ [19]. Using the configuration-interaction plus core-polarization (CICP) method [20], Spelsberg and Meyer [21] have calculated the

\footnotetext{
*junyi.zhang@kaust.edu.sa

†udo.schwingenschlogl@kaust.edu.sa
}

coefficients $C_{6}, C_{8}$, and $C_{10}$ for the interaction between $\mathrm{He}\left(2^{1,3} S\right)$ and the ground states of $\mathrm{Li}, \mathrm{Na}$, and $\mathrm{K}$.

In the present work, we compute the dispersion coefficients for the long-range interaction of the first four excited states of $\mathrm{He}$, i.e., $\mathrm{He}\left(2^{1,3} S\right)$ and $\mathrm{He}\left(2^{1,3} P\right)$, with the low-lying states of the alkali-metal atoms $\mathrm{Li}, \mathrm{Na}, \mathrm{K}$, and $\mathrm{Rb}$ by summing over the reduced matrix elements of the multipole transition operators [22-24]. For the symmetries of diatomic molecules we adopt the notations of Ref. [24]. Atomic units are used throughout the following sections.

A systematic formalism has been presented for the calculations of the long-range interaction between two heteronuclear atoms in arbitrary atomic states in Ref. [24], treating the interaction as a perturbation to the isolated atoms. In general, the long-range interaction between two heteronuclear atoms can be written in the form

$$
V(R)=-\sum_{s=1}^{\infty} \frac{C_{2 s+4}}{R^{2 s+4}}-\sum_{s=1}^{\ell_{a}+\ell_{b}-1} \frac{C_{2 s+3}}{R^{2 s+3}}-\cdots
$$

where $\ell_{a}$ and $\ell_{b}$ represent the quantum numbers of orbital angular momenta of atoms $A$ and $B$, respectively; $R$ is the distance between the two atoms; and $C_{n}$ are the dispersion coefficients. The first term arises from the second order correction to the energy and is always present. The second term (the first-order correction) occurs only if each atom is in a state with nonzero angular momentum.

The dispersion coefficients are evaluated for diatomic molecular states according to the nondegenerate and degenerate perturbation theories. For a simple nondegenerate system where both atoms $A$ and $B$ are spherically symmetric, for example, $C_{6}$ can be represented in the form

$$
\begin{aligned}
C_{6}= & \sum_{n_{a} n_{b}} \frac{2\left|\left\langle\psi_{0_{a}}\left\|\sum_{i} r_{i} \mathbf{C}^{1}\left(\hat{\mathbf{r}}_{i}\right)\right\| \psi_{n_{a}}\right\rangle\right|^{2}}{3} \\
& \times \frac{\left|\left\langle\psi_{0_{b}}\left\|\sum_{j} r_{j} \mathbf{C}^{1}\left(\hat{\mathbf{r}}_{j}\right)\right\| \psi_{n_{b}}\right\rangle\right|^{2}}{E_{n_{a}}+E_{n_{b}}-E_{0_{a}}-E_{0_{b}}}
\end{aligned}
$$


where $\mathbf{C}^{1}(\hat{\mathbf{r}})$ is the spherical tensor of rank $1 ; \psi_{0_{a}}$ and $\psi_{n_{a}}$ are the wave function of the initial state and the $n_{a}$ th intermediate eigenfunction for the atom $A$, respectively; $E_{0_{a}}$ and $E_{n_{a}}$ are their corresponding eigenenergies; the sum $i$ runs over all the electrons in the atom $A$; the definitions of symbols $\psi_{0_{b}}$, $\psi_{n_{b}}, E_{0_{b}}, E_{n_{b}}$ and $j$ for the atom $B$ are similar to those of their counterpart symbols for the atom $A$. In the degenerate case, the zeroth-order wave functions are determined by diagonalizing the leading term of the first-order correction in the degenerate space. They are then used to calculate the dispersion coefficients by summing over intermediate states represented by atomic physical states and pseudostates.

For atomic He and Li the energy spectra and reduced matrix elements of the multipole transition operators are the same as those used for calculating the dispersion coefficients for the low-lying states of He and Li [25-31]. They were calculated using Hylleraas basis functions. For a two-electron system, the basis functions have the form

$$
r_{1}^{i} r_{2}^{j} r_{12}^{k} e^{-\alpha r_{1}-\beta r_{2}} \mathcal{Y}_{\ell_{1} \ell_{2}}^{L M}\left(\hat{\mathbf{r}}_{1}, \hat{\mathbf{r}}_{2}\right),
$$

TABLE I. Dispersion coefficients for the interaction of $\mathrm{He}\left(2^{1,3} S\right)$ with the $n^{2} S$ ground states of the alkali-metal atoms. Numbers in square brackets in the second, third, and fourth columns denote powers of 10 .

\begin{tabular}{|c|c|c|c|}
\hline System & $C_{6}$ (a.u.) & $C_{8}$ (a.u.) & $C_{10}$ (a.u.) \\
\hline $\operatorname{LiHe}\left(2{ }^{1} S\right)^{2} \Sigma$ & $3.504[3]$ & $2.633[5]$ & $3.018[7]$ \\
\hline Hylleraas & $3.502[3]$ & $2.632[5]$ & $3.016[7]$ \\
\hline Ref. [18] & $3.500[3]$ & & \\
\hline Ref. [21] & $3.495[3]$ & $2.627[5]$ & $2.990[7]$ \\
\hline No core effect & $3.499[3]$ & $2.628[5]$ & $3.013[7]$ \\
\hline $\mathrm{NaHe}\left(2{ }^{1} S\right)^{2} \Sigma$ & $3.598[3]$ & $3.019[5]$ & $3.606[7]$ \\
\hline Ref. [18] & $3.660[3]$ & & \\
\hline Ref. [21] & $3.574[3]$ & $2.997[5]$ & $3.558[7]$ \\
\hline No core effect & $3.567[3]$ & $2.994[5]$ & $3.579[7]$ \\
\hline $\operatorname{KHe}\left(2{ }^{1} S\right)^{2} \Sigma$ & $5.984[3]$ & $5.805[5]$ & $7.588[7]$ \\
\hline Ref. [18] & $5.940[3]$ & & \\
\hline Ref. [21] & $5.845[3]$ & $5.690[5]$ & $7.600[7]$ \\
\hline No core effect & $5.816[3]$ & $5.668[5]$ & 7.439[7] \\
\hline $\operatorname{RbHe}\left(2{ }^{1} S\right)^{2} \Sigma$ & $6.509[3]$ & $6.846[5]$ & $9.207[7]$ \\
\hline Ref. [18] & $6.440[3]$ & & \\
\hline No core effect & $6.235[3]$ & $6.614[5]$ & $8.954[7]$ \\
\hline $\operatorname{LiHe}\left(2{ }^{3} S\right)^{2,4} \Sigma$ & $2.090[3]$ & $1.326[5]$ & $1.280[7]$ \\
\hline Hylleraas & $2.089[3]$ & $1.325[5]$ & $1.279[7]$ \\
\hline Ref. [18] & $2.090[3]$ & & \\
\hline Ref. [21] & $2.083[3]$ & $1.321[5]$ & $1.272[7]$ \\
\hline No core effect & $2.086[3]$ & $1.323[5]$ & $1.278[7]$ \\
\hline $\mathrm{NaHe}\left(2{ }^{3} S\right)^{2,4} \Sigma$ & $2.178[3]$ & $1.553[5]$ & $1.570[7]$ \\
\hline Ref. [18] & $2.220[3]$ & & \\
\hline Ref. [21] & $2.159[3]$ & $1.540[5]$ & $1.553[7]$ \\
\hline No core effect & $2.157[3]$ & $1.540[5]$ & $1.560[7]$ \\
\hline $\operatorname{KHe}\left(2{ }^{3} S\right)^{2,4} \Sigma$ & $3.523[3]$ & $3.055[5]$ & $3.485[7]$ \\
\hline Ref. [18] & $3.480[3]$ & & \\
\hline Ref. [21] & $3.419[3]$ & $2.993[5]$ & $3.534[7]$ \\
\hline No core effect & $3.406[3]$ & $2.983[5]$ & $3.426[7]$ \\
\hline $\operatorname{RbHe}\left(2^{3} S\right)^{2,4} \Sigma$ & $3.832[3]$ & $3.634[5]$ & 4.294[7] \\
\hline Ref. [18] & $3.760[3]$ & & \\
\hline No core effect & $3.641[3]$ & $3.512[5]$ & 4.191[7] \\
\hline
\end{tabular}

TABLE II. Dispersion coefficients for the interaction of $\mathrm{He}\left(2^{1,3} S\right)$ with the first ${ }^{2} P$ states of the alkali-metal atoms. Numbers in square brackets denote powers of 10 .

\begin{tabular}{lrll}
\hline \hline System & $C_{6}$ (a.u.) & $C_{8}$ (a.u.) & $C_{10}$ (a.u.) \\
\hline LiHe $\left(2{ }^{1} S\right)^{2} \Sigma$ & $2.048[3]$ & $2.483[6]$ & $2.600[8]$ \\
Hylleraas & $2.050[3]$ & $2.481[6]$ & \\
LiHe $\left(2{ }^{1} S\right)^{2} \Pi$ & $2.663[3]$ & $5.222[5]$ & $3.298[7]$ \\
Hylleraas & $2.663[3]$ & $5.219[5]$ & \\
$\mathrm{NaHe}\left(2{ }^{1} S\right)^{2} \Sigma$ & $8.845[3]$ & $5.392[6]$ & $6.368[8]$ \\
$\mathrm{NaHe}\left(2{ }^{1} S\right)^{2} \Pi$ & $6.364[3]$ & $9.699[5]$ & $5.672[7]$ \\
$\mathrm{KHe}\left(2{ }^{1} S\right)^{2} \Sigma$ & $9.756[3]$ & $7.974[6]$ & $1.219[9]$ \\
$\mathrm{KHe}\left(2{ }^{1} S\right)^{2} \Pi$ & $9.242[3]$ & $9.847[5]$ & $7.951[7]$ \\
$\operatorname{RbHe}\left(2{ }^{1} S\right)^{2} \Sigma$ & $1.298[4]$ & $1.012[7]$ & $1.652[9]$ \\
$\operatorname{RbHe}\left(2{ }^{1} S\right)^{2} \Pi$ & $1.174[4]$ & $1.078[6]$ & $9.601[7]$ \\
$\operatorname{LiHe}\left(2{ }^{3} S\right)^{2,4} \Sigma$ & $-2.190[3]$ & $1.063[6]$ & $1.230[8]$ \\
$\mathrm{Hylleraas}$ & $-2.187[3]$ & $1.062[6]$ & \\
$\mathrm{LiHe}\left(2{ }^{3} S\right)^{2,4} \Pi$ & $7.605[2]$ & $1.571[5]$ & $1.034[7]$ \\
$\mathrm{Hylleraas}$ & $7.611[2]$ & $1.570[5]$ & \\
$\mathrm{NaHe}\left(2{ }^{3} S\right)^{2,4} \Sigma$ & $1.969[3]$ & $2.252[6]$ & $3.142[8]$ \\
$\mathrm{NaHe}\left(2{ }^{3} S\right)^{2,4} \Pi$ & $2.919[3]$ & $2.535[5]$ & $1.804[7]$ \\
$\mathrm{KHe}\left(2{ }^{3} S\right)^{2,4} \Sigma$ & $-3.189[3]$ & $3.776[6]$ & $6.189[8]$ \\
$\mathrm{KHe}\left(2{ }^{3} S\right)^{2,4} \Pi$ & $2.995[3]$ & $3.147[5]$ & $2.696[7]$ \\
$\operatorname{RbHe}\left(2{ }^{3} S\right)^{2,4} \Sigma$ & $-3.007[3]$ & $4.801[6]$ & $8.430[8]$ \\
$\operatorname{RbHe}\left(2{ }^{3} S\right)^{2,4} \Pi$ & $3.851[3]$ & $3.491[5]$ & $3.297[7]$ \\
\hline \hline
\end{tabular}

where $\mathcal{Y}_{\ell_{1} \ell_{2}}^{L M}\left(\hat{\mathbf{r}}_{1}, \hat{\mathbf{r}}_{2}\right)$ are the coupled spherical harmonics. Except for some truncations made to avoid near linear dependence, all terms with $i+j+k \leqslant \Omega$ are included, where $\Omega$ is an integer. For the $\mathrm{Li}$ atom the basis functions have a similar form:

$$
r_{1}^{i} r_{2}^{j} r_{3}^{k} r_{12}^{m} r_{13}^{n} r_{23}^{p} e^{-\alpha r_{1}-\beta r_{2}-\gamma r_{3}} \mathcal{Y}_{\left(\ell_{1} \ell_{2}\right) \ell_{12}, \ell_{3}}^{L M}\left(\hat{\mathbf{r}}_{1}, \hat{\mathbf{r}}_{2}, \hat{\mathbf{r}}_{3}\right) .
$$

The nonlinear parameters $\alpha, \beta$, and $\gamma$ are variationally optimized by the power method.

The reduced matrix elements of the transition operators and energy spectra for the alkali-metal atoms have been used for calculating the dispersion coefficients for the interaction with ground-state $\mathrm{H}$ and $\mathrm{He}$ [24]. The spectra of the valence electrons have been generated by the CICP method with large mixed Laguerre-type and Slater-type orbital basis sets. Moreover, the core effect on the valence electrons has been represented by a semiempirical polarization potential, and the contribution of the core excitations to the dispersion coefficients has been estimated by approximate oscillator strength distributions. For details on the calculations for alkali-metal atoms the reader is referred to Refs. [24,32-37].

Table I presents the dispersion coefficients for the interaction of $\operatorname{He}\left(2^{1,3} S\right)$ with the ground-state alkali-metal atoms. Table II addresses the interaction between $\operatorname{He}\left(2^{1,3} S\right)$ and the first ${ }^{2} P$ excited states of the alkali-metal atoms, i.e., $\operatorname{Li}\left(2^{2} P\right)$, $\mathrm{Na}\left(3^{2} P\right), \mathrm{K}\left(4^{2} P\right)$, and $\mathrm{Rb}\left(5^{2} P\right)$ whereas Table III refers to the interaction between $\mathrm{He}\left(2^{1,3} P\right)$ and the ground-state alkalimetal atoms. Dispersion coefficients $C_{n}$ for the interaction between $\operatorname{He}\left(2^{1,3} S\right)$ and the first ${ }^{2} S$ and ${ }^{2} D$ excited states of the alkali-metal atoms and those for the interaction between $\mathrm{He}\left(2^{1,3} P\right)$ and the first ${ }^{2} P$ excited states of the alkali-metal atoms are listed in the Supplemental Material [38].

For the Li atom we calculate the dispersion coefficients using both the CICP transition data and the transition data 
TABLE III. Dispersion coefficients for the interaction of $\mathrm{He}\left(2^{1,3} P\right)$ with the ground states of the alkali-metal atoms. Numbers in square brackets denote powers of 10 .

\begin{tabular}{llll}
\hline \hline System & $C_{6}$ (a.u.) & $C_{8}$ (a.u.) & $C_{10}$ (a.u.) \\
\hline $\operatorname{LiHe}\left(2{ }^{1} P\right)^{2} \Sigma$ & $6.123[3]$ & $7.851[5]$ & $1.019[8]$ \\
Hylleraas & $6.120[3]$ & $7.847[5]$ & $1.018[8]$ \\
$\operatorname{LiHe}\left(2{ }^{1} P\right)^{2} \Pi$ & $2.605[3]$ & $8.148[4]$ & $5.676[6]$ \\
Hylleraas & $2.604[3]$ & $8.143[4]$ & $5.672[6]$ \\
$\operatorname{NaHe}\left(2{ }^{1} P\right)^{2} \Sigma$ & $5.981[3]$ & $8.510[5]$ & $1.179[8]$ \\
$\mathrm{NaHe}\left(2{ }^{1} P\right)^{2} \Pi$ & $2.633[3]$ & $1.013[5]$ & $7.649[6]$ \\
$\mathrm{KHe}\left(2{ }^{1} P\right)^{2} \Sigma$ & $1.114[4]$ & $1.543[6]$ & $2.330[8]$ \\
$\mathrm{KHe}\left(2{ }^{1} P\right)^{2} \Pi$ & $4.575[3]$ & $2.518[5]$ & $2.200[7]$ \\
$\operatorname{RbHe}\left(2{ }^{1} P\right)^{2} \Sigma$ & $1.223[4]$ & $1.768[6]$ & $2.772[8]$ \\
$\operatorname{RbHe}\left(2{ }^{1} P\right)^{2} \Pi$ & $5.008[3]$ & $3.175[5]$ & $2.887[7]$ \\
$\operatorname{LiHe}\left(2{ }^{3} P\right)^{2,4} \Sigma$ & $7.153[3]$ & $5.617[5]$ & $6.620[7]$ \\
$\mathrm{Hylleraas}$ & $7.148[3]$ & $5.614[5]$ & $6.616[7]$ \\
$\mathrm{LiHe}\left(2{ }^{3} P\right)^{2,4} \Pi$ & $2.609[3]$ & $6.790[4]$ & $4.464[6]$ \\
$\mathrm{Hylleraas}$ & $2.607[3]$ & $6.785[4]$ & $4.461[6]$ \\
$\mathrm{NaHe}\left(2{ }^{3} P\right)^{2,4} \Sigma$ & $6.318[3]$ & $6.167[5]$ & $7.754[7]$ \\
$\mathrm{NaHe}\left(2{ }^{3} P\right)^{2,4} \Pi$ & $2.452[3]$ & $8.484[4]$ & $6.053[6]$ \\
$\mathrm{KHe}\left(2{ }^{3} P\right)^{2,4} \Sigma$ & $1.534[4]$ & $1.166[6]$ & $1.571[8]$ \\
$\operatorname{KHe}\left(2{ }^{3} P\right)^{2,4} \Pi$ & $5.198[3]$ & $2.193[5]$ & $1.779[7]$ \\
$\operatorname{RbHe}\left(2{ }^{3} P\right)^{2,4} \Sigma$ & $1.738[4]$ & $1.371[6]$ & $1.887[8]$ \\
$\operatorname{RbHe}\left(2{ }^{3} P\right)^{2,4} \Pi$ & $5.833[3]$ & $2.832[5]$ & $2.349[7]$ \\
\hline \hline
\end{tabular}

computed by the Hylleraas-type basis functions. For the $\operatorname{Li}\left(2{ }^{2} S\right)$ and $\operatorname{Li}\left(3{ }^{2} S\right)$ states the coefficients $C_{6}, C_{8}$, and $C_{10}$ are computed using the correlated basis sets. For the $\operatorname{Li}\left(2^{2} P\right)$ and $\mathrm{Li}\left(3^{2} D\right)$ states the coefficients up to $C_{8}$ are computed using the Hylleraas-type basis sets, because only the intermediate states related to the corresponding dipole and quadrupole transitions have been generated with the Hylleraas-type basis sets. The results of Dalgarno and Victor [18] and Spelsberg and Meyer [21] are also included in Table I for comparison. These four calculations agree within $0.3 \%$ for the $\operatorname{Li}\left(2{ }^{2} S\right)$ ground state. For the $\mathrm{He}\left(2{ }^{1,3} S\right)-\mathrm{Na}\left(3^{2} S\right)$ system the $C_{6}$ coefficient calculated by Dalgarno and Victor [18] is $2 \%$ larger than our result. Moreover, their $C_{6}$ values are $1 \%$ smaller than our values for the systems $\mathrm{He}\left(2{ }^{1,3} S\right)-\mathrm{K}\left(4{ }^{2} S\right)$ and $\mathrm{He}\left(2{ }^{1} S\right)-\mathrm{Rb}\left(5{ }^{2} S\right)$, and $2 \%$ smaller for the $\operatorname{He}\left(2{ }^{3} S\right)-\operatorname{Rb}\left(5^{2} S\right)$ system.

The effects of core excitations on the dispersion coefficients are ignored in the calculations of Spelsberg and Meyer [21]. To enable a comparison, we also list in Table I our $C_{n}$ values which exclude contributions of the core excitations. The discrepancy between the two calculations without core effects is less than $0.5 \%$ except for the $C_{10}$ values for the $\operatorname{He}\left(2{ }^{1,3} S\right)-\mathrm{K}\left(4^{3} S\right)$ system. The $C_{10}$ values of Spelsberg and Meyer are 2-3\% larger than our results for the $\operatorname{He}\left(2{ }^{1,3} S\right)-\mathrm{K}\left(4^{3} S\right)$ system. This means that the octupole contributions are overestimated in their calculations. The overestimation is confirmed by comparing their values for the static multipole polarizabilities with other accurate calculations [32,33,39-43] (see Table I in the Supplemental Material [38]). For the static dipole polarizability of the ground-state $\mathrm{K}$ the spread of all four calculations is within $2.5 \%$, and the four quadrupole polarizabilities are almost the same. However, the static octupole polarizability of Spelsberg and Meyer $\left(1.914 \times 10^{5}\right.$ a.u. $)$ is $7.7 \%$ larger than the value obtained by the other three calculations (the CICP data set, the one-electron model potential approach by Marinescu et al. [40], and the relativistic many-body perturbation theory by Porsev and Derevianko [43]). It is also demonstrated that the core effect becomes more important for heavier atoms.

The uncertainty of the present calculations can be estimated from the uncertainties of the dispersion coefficients for interaction of the alkali-metal atoms with the ground states of $\mathrm{H}$ and $\mathrm{He}$ [24]. For the systems $\mathrm{He}\left(2^{1,3} S\right)-\mathrm{Li}\left(2^{2} S\right)$, $\mathrm{He}\left(2^{1,3} S\right)-\operatorname{Li}\left(2^{2} P\right)$, and $\mathrm{He}\left(2{ }^{3} S\right)-\operatorname{Li}\left(3^{2} D\right)[38]$ the accuracy of the calculations is $0.1 \%$. For the system $\mathrm{He}\left(2{ }^{1} S\right)-\mathrm{Li}\left(3^{2} D\right)$ [38], the $C_{6}$ values of the Hylleraas and CICP methods deviate by less than $0.1 \%$ and the discrepancy of the $C_{8}$ values is $0.3 \%$. For the ${ }^{2} \Sigma$ state of the system $\operatorname{He}\left(2^{1} P\right)-\operatorname{Li}\left(3{ }^{2} S\right)[38]$ the discrepancy between the two methods is $0.7 \%$ for $C_{8}$. For both the ${ }^{2} \Sigma$ and ${ }^{2} \Pi$ states of the system $\operatorname{He}\left(2{ }^{3} P\right)-\operatorname{Li}\left(3{ }^{2} S\right)$ [38] the discrepancy of the $C_{8}$ and $C_{10}$ values between the two methods is $2 \%$. For the system $\operatorname{He}\left(2^{1} P\right)-\operatorname{Li}\left(2^{2} P\right)$ [38] the two methods agree within $0.5 \%$. The $C_{6}$ coefficient deviates between the two methods by $90 \%$ for the second ${ }^{2,4} \Sigma^{+}$state of the $\operatorname{He}\left(2^{3} P\right)$ $\mathrm{Li}(2 P)$ system [38]. The reason for this large difference is that the negative contribution of $-1.530 \times 10^{4}$ a.u. of the dipole transition pairs $\mathrm{He}\left(2{ }^{3} P\right)-\mathrm{He}\left(2{ }^{3} S\right)$ and $\mathrm{Li}\left(2^{2} P\right)-\mathrm{Li}\left(2{ }^{2} S\right)$ as well as $\mathrm{He}\left(2{ }^{3} P\right)-\mathrm{He}\left(3^{3} S\right)$ and $\operatorname{Li}\left(2^{2} P\right)-\operatorname{Li}\left(2{ }^{2} S\right)$ almost cancels the positive contribution of $1.535 \times 10^{4}$ a.u. of all other dipole transition pairs. No such strong cancellation occurs for other dispersion coefficients. Another unexpected feature is that the $C_{6}$ coefficient is about 19 times smaller than the corresponding $C_{5}$ coefficient. In summary, the results from the Hylleraas-type calculations are recommended rather than those from the CICP calculations.

For the long-range interaction between $\mathrm{He}$ and other alkali-metal atoms, the uncertainties are $1-3 \%$ for $C_{5}, 1-5 \%$ for $C_{6}$, and $1-10 \%$ for $C_{8}$ and $C_{10}$. Generally speaking, the uncertainties of the dispersion coefficients for the ground states of the alkali-metal atoms are smaller than those for the excited states, especially in the cases where the He atom is in one of the $\operatorname{He}\left(2^{1,3} P\right)$ states.

In conclusion, dispersion coefficients have been calculated for the long-range interaction of the first four excited states of He, i.e., $\operatorname{He}\left(2^{1,3} S\right)$ and $\operatorname{He}\left(2^{1,3} P\right)$, with the low-lying states of the alkali-metal atoms $\mathrm{Li}, \mathrm{Na}, \mathrm{K}$, and $\mathrm{Rb}$ by summing over the reduced matrix elements of the multipole transition operators [22-24]. For He and Li atoms the reduced matrix elements have been previously generated with Hylleraas-type basis functions [25-31]. For the alkali-metal atoms the transition arrays of the valence electrons have been previously computed by the CICP method, where the effect of core excitations has been taken into account by approximately constructing the oscillator strength distributions of the atomic cores [24,32-37]. For systems involving a $\mathrm{He}$ metastable state $\operatorname{He}\left(2^{1,3} S\right)$ and the ground state of an alkali-metal atom, our results are more accurate than previously published values [18,21]. Dispersion coefficients for systems involving a $\mathrm{He}\left(2^{1,3} P\right)$ state or excited state of an alkali-metal atom have been computed. These coefficients enable the construction of accurate long-range potentials for the corresponding atom-atom collisions.

The authors would like to thank Jim Mitroy for allowing them to use his CICP transition spectra of the alkali-metal atoms. Z.-C. Yan was supported by NSERC of Canada, 
by the Canadian computing facilities of SHARCnet and ACEnet, and in part by the Chinese Academy of Sciences CAS/SAFEA International Partnership Program for Creative
Research Teams. L.-Y. Tang was supported by NNSF of China under Grant No. 11104323 and by the National Basic Research Program of China under Grant No. 2012CB821305.
[1] G. Modugno, G. Ferrari, G. Roati, R. J. Brecha, A. Simoni, and I. M, Science 294, 1320 (2001).

[2] G. Roati, F. Riboli, G. Modugno, and M. Inguscio, Phys. Rev. Lett. 89, 150403 (2002).

[3] M. Anderlini, E. Courtade, M. Cristiani, D. Cossart, D. Ciampini, C. Sias, O. Morsch, and E. Arimondo, Phys. Rev. A 71, 061401 (2005).

[4] J. P. Shaffer, W. Chalupczak, and N. P. Bigelow, Phys. Rev. Lett. 82, 1124 (1999).

[5] D. Wang, J. Qi, M. F. Stone, O. Nikolayeva, H. Wang, B. Hattaway, S. D. Gensemer, P. L. Gould, E. E. Eyler, and W. C. Stwalley, Phys. Rev. Lett. 93, 243005 (2004).

[6] K.-K. Ni, S. Ospelkaus, M. H. G. de Miranda, A. Peer, B. Neyenhuis, J. J. Zirbel, S. Kotochigova, P. S. Julienne, D. S. Jin, and J. Ye, Science 322, 231 (2008).

[7] D. DeMille, Phys. Rev. Lett. 88, 067901 (2002).

[8] A. Micheli, G. K. Brennen, and P. Zoller, Nat. Phys. 2, 341 (2006).

[9] M. W. Mancini, A. R. L. Caires, G. D. Telles, V. S. Bagnato, and L. G. Marcassa, Eur. Phys. J. D 30, 105 (2004).

[10] S. Hensler, A. Griesmaier, J. Werner, A. Görlitz, and T. Pfau, J. Mod. Opt. 51, 1807 (2004).

[11] H. C. Busch, M. K. Shaffer, E. M. Ahmed, and C. I. Sukenik, Phys. Rev. A 73, 023406 (2006).

[12] W. Vassen, C. Cohen-Tannoudji, M. Leduc, D. Boiron, C. I. Westbrook, A. Truscott, K. Baldwin, G. Birkl, P. Cancio, and M. Trippenbach, Rev. Mod. Phys. 84, 175 (2012).

[13] L. J. Byron, R. G. Dall, and A. G. Truscott, Phys. Rev. A 81, 013405 (2010).

[14] L. J. Byron, R. G. Dall, W. Rugway, and A. G. Truscott, New J. Phys. 12, 013004 (2010).

[15] M.-W. Ruf, A. J. Yencha, and H. Hotop, Z. Phys. D 5, 9 (1987).

[16] K. L. Bell, A. Dalgarno, and A. E. Kingston, J. Phys. B 1, 18 (1968).

[17] H. B. G. Casimir and D. Polder, Phys. Rev. 73, 360 (1948).

[18] A. D. Dalgarno and G. A. Victor, J. Chem. Phys. 49, 1982 (1968).

[19] A. Dalgarno and W. D. Davison, Mol. Phys. 13, 479 (1967).

[20] W. Müller, J. Flesch, and W. Meyer, J. Chem. Phys. 80, 3297 (1984).
[21] D. Spelsberg and W. Meyer, J. Chem. Phys. 99, 8351 (1993).

[22] A. Dalgarno and W. D. Davison, Adv. At. Mol. Phys. 2, 1 (1966).

[23] A. Dalgarno, Adv. Chem. Phys. 12, 143 (1967).

[24] J. Y. Zhang and J. Mitroy, Phys. Rev. A 76, 022705 (2007).

[25] J. Y. Zhang, Z. C. Yan, D. Vrinceanu, and H. R. Sadeghpour, Phys. Rev. A 71, 032712 (2005).

[26] J. Y. Zhang, Z. C. Yan, D. Vrinceanu, J. F. Babb, and H. R. Sadeghpour, Phys. Rev. A 73, 022710 (2006).

[27] J. Y. Zhang, Z. C. Yan, D. Vrinceanu, J. F. Babb, and H. R. Sadeghpour, Phys. Rev. A 74, 014704 (2006).

[28] J. Y. Zhang, Z. C. Yan, D. Vrinceanu, J. F. Babb, and H. R. Sadeghpour, Phys. Rev. A 76, 012723 (2007).

[29] L.-Y. Tang, Z.-C. Yan, T.-Y. Shi, and J. F. Babb, Phys. Rev. A 79, 062712 (2009).

[30] L.-Y. Tang, Z.-C. Yan, T.-Y. Shi, and J. Mitroy, Phys. Rev. A 81, 042521 (2010).

[31] L.-Y. Tang, J.-Y. Zhang, Z.-C. Yan, T.-Y. Shi, and J. Mitroy, J. Chem. Phys. 133, 104306 (2010).

[32] J. Mitroy and M. W. J. Bromley, Phys. Rev. A 68, 052714 (2003).

[33] J. Mitroy and M. W. J. Bromley, Phys. Rev. A 71, 019902(E) (2005); 71, 019903(E) (2005).

[34] M. W. J. Bromley and J. Mitroy, Phys. Rev. A 65, 012505 (2001).

[35] M. W. J. Bromley and J. Mitroy, Phys. Rev. A 65, 062505 (2002).

[36] J. Mitroy and M. W. J. Bromley, Phys. Rev. A 70, 052503 (2004).

[37] J. Mitroy and J. Y. Zhang, Phys. Rev. A 76, 032706 (2007).

[38] See Supplemental Material at http://link.aps.org/supplemental/ 10.1103/PhysRevA.86.064701 for the long-range interaction coefficients for other pairs of excited He and alkali-metal atoms.

[39] D. Spelsberg, T. Lorenz, and W. Meyer, J. Chem. Phys. 99, 7845 (1993).

[40] M. Marinescu, H. R. Sadeghpour, and A. Dalgarno, Phys. Rev. A 49, 982 (1994).

[41] A. Derevianko, W. R. Johnson, M. S. Safronova, and J. F. Babb, Phys. Rev. Lett. 82, 3589 (1999).

[42] M. S. Safronova, W. R. Johnson, and A. Derevianko, Phys. Rev. A 60, 4476 (1999).

[43] S. G. Porsev and A. Derevianko, J. Chem. Phys. 119, 844 (2003). 